\title{
ON A THEOREM BY J. L. WALSH CONCERNING THE MODULI OF ROOTS OF ALGEBRAIC EQUATIONS
}

\author{
ALEXANDER OSTROWSKI
}

In $1881 \mathrm{~A}$. E. Pellet published ${ }^{1}$ the following very useful theorem:

If the polynomial

$$
\begin{aligned}
F(z) \equiv\left|a_{0}\right|+\left|a_{1}\right| z+\left|a_{2}\right| z^{2}+\cdots+\left|a_{k-1}\right| z^{k-1} \\
-\left|a_{k}\right| z^{k}+\left|a_{k+1}\right| z^{k+1}+\cdots+\left|a_{n}\right| z^{n}, \\
0<k<n, a_{0} a_{n} \neq 0,
\end{aligned}
$$

has two positive roots $x_{1}$ and $x_{2}\left(x_{1}<x_{2}\right)$, then the polynomial

$$
f(z) \equiv a_{0}+a_{1} z+a_{2} z^{2}+\cdots+a_{n} z^{n}
$$

has no roots in the annulus $x_{1}<|z|<x_{2}$ and precisely $k$ roots in the circle $|z| \leqq x_{1}$.

While Pellet's proof for his theorem utilizes the theorem of Rouché, J. L. Walsh published in $1924^{2}$ another more direct proof and established in the same memoir a sort of converse of Pellet's theorem. To formulate his result, consider the set $\mathfrak{F}$ of all polynomials

$$
f(z) \equiv a_{0}+a_{1} z+a_{2} z^{2}+\cdots+a_{n} z^{n}
$$

which correspond to given moduli of coefficients. All polynomials of $\mathfrak{F}$ are obtained from one of them, $f(z)$, if the factors $\epsilon_{0}, \epsilon_{1}, \cdots, \epsilon_{n}$ in the expression

$$
\epsilon_{0} a_{0}+\epsilon_{1} a_{1} z+\epsilon_{2} a_{2} z^{2}+\cdots+\epsilon_{n} a_{n} z^{n}
$$

assume independently all values of modulus 1 . Let $\mathfrak{M}$ be the set of roots of all polynomials in $\mathfrak{F}$. It is immediately seen that if $\mathfrak{M}$ contains a number $\alpha$ it also contains all numbers with the modulus $|\alpha|$.

It was proved by Cauchy that all roots of (4) lie on or within the circle $|z|=y_{1}$, where $y_{1}$ is the single positive root of the polynomial

$$
\left|a_{0}\right|+\left|a_{1}\right| z+\cdots+\left|a_{n-1}\right| z^{n-1}-\left|a_{n}\right| z^{n}
$$

and that all roots of (4) lie on or are exterior to the circle $|z|=y_{2}$, where $y_{2}$ is the single positive root of the polynomial

${ }^{1}$ A. E. Pellet: Sur un mode de séparation des racines des équations et la formule de Lagrange, Bulletin des Sciences Mathématiques, (2), vol. 5 (1881), pp. 393-395.

${ }^{2} \mathrm{~J}$. L. Walsh: On Pellet's theorem concerning the roots of a polynomial, Annals of Mathematics, vol. 26 (1924), pp. 59-64. 


$$
\left|a_{0}\right|-\left|a_{1}\right| z-\cdots-\left|a_{n}\right| z^{n}
$$

Since the numbers $y_{1}$ and $y_{2}$ obviously belong to $\mathfrak{M}$, it follows that $\mathfrak{M}$ lies in the closed annulus $y_{2} \leqq|z| \leqq y_{1}$.

Suppose now that $a>0$ is not contained in $\mathfrak{M}$. Then, if $C$ is the circle $|z|=a$, all polynomials of $\mathfrak{F}$ contain the same number $k$ of roots within $C$. Indeed, if we vary continuously the factors $\epsilon_{\nu}$ in the representation (4), the roots of (4) vary also continuously and their number within $C$ remains the same since none of them is able to pass $C$.

Now the theorem of Walsh runs as follows:

Any positive a that is not contained in $\mathfrak{M}$, and for which the number of roots of (4) within the circle $|z|=a$ is precisely $k(0<k<n)$, is contained between the two positive roots of the polynomial (1), that is to say:

$$
\begin{aligned}
& \left|a_{k}\right| a^{k}>\left(\left|a_{0}\right|+\cdots+\left|a_{k-1}\right| a^{k-1}\right) \\
& \quad+\left(\left|a_{k+1}\right| a^{k+1}+\cdots+\left|a_{n}\right| a^{n}\right) .
\end{aligned}
$$

As the proof given by Walsh of this important result is not complete, ${ }^{3}$ we give in what follows another proof proceeding on different lines.

Suppose that, contrary to (5),

$$
\left|a_{k}\right| a^{k} \leqq \sum_{\nu=0, \nu \neq k}^{n}\left|a_{\nu}\right| a^{\nu}
$$

then, as $a$ is not contained in $\mathfrak{M}$, we have even

$$
\left|a_{k}\right| a^{k}<\sum_{\nu=0, \nu \neq k}^{n}\left|a_{\nu}\right| a^{\nu} .
$$

On the other hand it follows from our hypothesis that if $\epsilon_{\nu}$ run independently through all constants of modulus 1 ,

$$
\sum_{\nu=0}^{n} \epsilon_{\nu} a_{\nu} a^{\nu} \neq 0
$$

and therefore

3 Walsh allows in his proof the roots of $f(z)$, which are in absolute value less than $a$, to vary continuously and monotonically (in absolute value) and to approach 0 . But during this variation the polynomial $f(z)$ does not necessarily remain in the set $\mathfrak{F}$ and the corresponding sets $\mathfrak{F}_{0}$ for the polynomials thus obtained could very well contain $a$ in the set of the roots, so that the expression (1) need not remain positive for $z=a$, as is assumed in Walsh's proof. 


$$
\left|a_{k}\right| a^{k} \neq\left|\sum_{\nu=0, \nu \neq k} \epsilon_{\nu} a_{\nu} a^{\nu}\right| .
$$

But from (7) and (9) it follows that for all values of $\epsilon_{\nu}$ in question

$$
\left|a_{k}\right| a^{k}<\left|\sum_{\nu=0, \nu \neq l_{i}}^{n} \epsilon_{\nu} a_{\nu} a^{\nu}\right| .
$$

For, if we have for a particular set of $\epsilon_{\nu}$-values, $\epsilon_{\nu}^{0}$ :

$$
\left|a_{k}\right| a^{k}>\left|\sum_{\nu=0, \nu \neq k}^{n} \epsilon_{\nu}^{0} a_{\nu} a^{\nu}\right|,
$$

we see from ( 7$)$ and (11), that the right-hand side of (10) becomes equal to $\left|a_{k}\right| a^{k}$ for some other set of $\epsilon_{\nu}$, contrary to (9).

Now it follows from (10) that the polynomials

$$
f^{*}(z)=\sum_{\nu=0, \nu \neq k}^{n} \epsilon_{\nu} a_{\nu} z^{\nu}
$$

have no roots at $a$ and therefore no roots on the circle $C$. On the other hand, we have obviously by (10) everywhere on $C:\left|f^{*}(z)\right|>\left|a_{k} z^{k}\right|$. Hence, by the theorem of Rouché, since $f^{*}(z)-a_{k} z^{k}$ has exactly $k$ roots inside $C$, the same is true for any polynomial $f^{*}(z)$.

The result arrived at may be announced in the following form:

If $\epsilon_{0}, \cdots, \epsilon_{k-1}, \epsilon_{k+1}, \cdots, \epsilon_{n}$ run independently through all values of modulus 1 , let

$$
\phi(z)=\sum_{\nu=0}^{k-1} \epsilon_{\nu} a_{\nu} z^{\nu}, \quad \psi(z)=\sum_{\nu=k+1}^{n} \epsilon_{\nu} a_{\nu} z^{\nu}
$$

then the difference $\phi(z)-\psi(z)$ does not vanish on $C$ and has exactly $k$ roots inside $C$.

It follows, that in particular

$$
\left|\sum_{\nu=0}^{k-1} \epsilon_{\nu} a_{\nu} a^{\nu}\right| \neq\left|\sum_{\nu=k+1}^{n} \epsilon_{\nu} a_{\nu} a^{\nu}\right| .
$$

But then it is impossible that we have simultaneously for one particular set $\epsilon_{\nu}^{\prime}$ of $\epsilon_{\nu}$

$$
\left|\sum_{\nu=0}^{k-1} \epsilon_{\nu}^{\prime} a_{\nu} a^{\nu}\right|>\left|\sum_{\nu=k+1}^{n} \epsilon_{\nu}^{\prime} a_{\nu} a^{\nu}\right|
$$

and for another, $\epsilon_{\nu}{ }^{\prime \prime}$, 


$$
\left|\sum_{\nu=0}^{k-1} \epsilon_{\nu}^{\prime \prime} a_{\nu} a^{\nu}\right|<\left|\sum_{\nu=k+1}^{n} \epsilon_{\nu}^{\prime \prime} a_{\nu} a^{\nu}\right| .
$$

Hence only two cases are possible:

A. We have always

$$
\left|\sum_{\nu=0}^{k-1} \epsilon_{\nu} a_{\nu} a^{\nu}\right|>\left|\sum_{\nu=k+1}^{n} \epsilon_{\nu} a_{\nu} a^{\nu}\right|
$$

and therefore everywhere on $C:|\phi(z)|>|\psi(z)|$.

But then, by the theorem of Rouché, $\phi(z)$ has inside $C$ the same number of roots as $\phi(z)-\psi(z)$, that is $k$, and this is impossible, $\phi(z)$ being of degree $k-1$.

B. We have always

$$
\left|\sum_{\nu=0}^{k-1} \epsilon_{\nu} a_{\nu} a^{\nu}\right|<\left|\sum_{\nu=k+1}^{n} \epsilon_{\nu} a_{\nu} a^{\nu}\right|,
$$

and therefore everywhere on $C:|\phi(z)|<|\psi(z)|$.

But then $\psi(z)$ would have inside $C$ exactly $k$ roots, while $\psi(z)$ vanishes at $z=0$ with the multiplicity $k+1$ at least.

We see that (7) and (6) are impossible and the proof of (5) is completed.

The theorems of Pellet and Walsh dealt with in the preceding paragraphs allow us to describe immediately the set $\mathfrak{M}$.

Consider the $n+1$ equations:

$$
\begin{aligned}
\left|a_{n}\right| z^{n}-\sum_{\nu=0}^{n-1}\left|a_{\nu}\right| z^{\nu} & =0, \\
\sum_{\nu=k+1}^{n}\left|a_{\nu}\right| z^{\nu}-\left|a_{k}\right| z^{k}+\sum_{\nu=0}^{k-1}\left|a_{\nu}\right| z^{\nu} & =0, \quad k=1, \cdots, n-1, \\
\sum_{\nu=1}^{n}\left|a_{\nu}\right| z^{\nu}-\left|a_{0}\right| & =0 .
\end{aligned}
$$

If, as we will assume, $a_{0} a_{n} \neq 0$, each of the equations (13), (15) possesses one positive root $\rho$ resp. $\rho_{0}$, and we have $\rho_{0} \leqq \rho$ since $\rho$ is the exact upper and $\rho_{0}$ the exact lower limit for the moduli of $\mathfrak{M}$. As to the equations (14), every one of them possesses either two positive roots or exactly one double positive root or no positive roots at all. Strike out the equations (14) corresponding to the two last cases; each of the remaining equations (14) possesses two positive roots, $x_{k}, x_{k}^{\prime}$ 
$\left(x_{k}<x_{k}^{\prime}\right)$. Then we obtain $\mathfrak{M}$ by removing from the plane of $z$ the two circular domains $|z|<\rho_{0}$ and $|z|>\rho$ and all annuli $x_{k}<|z|<x_{k}^{\prime}$, corresponding to the equations (14) with two different positive roots.

It is then a consequence of Walsh's theorem that the different (open) intervals $\left(x_{k}, x_{k}^{\prime}\right)$ have no points in common and lie in the interval $\left(\rho_{0}, \rho\right)$; moreover, if for two of these intervals $\left(x_{k}, x_{k}^{\prime}\right)$, $\left(x_{m}, x_{m}^{\prime}\right)$ we have $k<m$, then we have certainly $x_{k}^{\prime} \leqq x_{m}$, and it is easily seen that we have even $x_{k}^{\prime}<x_{m}$.

As Walsh remarks, his proof of Pellet's theorem remains valid also in the case of a power-series and of its roots inside the circle of convergence. It is hardly necessary to remark that our proof of Walsh's theorem also applies mutatis mutandis to a power series, if we only consider its roots within the circle of convergence.

UNIVERSITY OF BASEL

\section{SOME EXCEPTIONAL VALUES OF THE LIMIT OF THE RATIO OF ARC TO CHORD}

\section{RICHARD COHN}

It was observed by $\mathrm{E}$. Kasner ${ }^{1}$ that in the complex euclidean plane the limiting value of the ratio of the arc of a curve to its chord, while one end point of the arc is fixed, and the other approaches it along the curve, is not always unity; but assumes for analytic curves tangent to a minimal line, a sequence of real values, $.94 \cdots, .86 \cdots, .80, \cdots$. These values are functions of the order of contact only, and approach zero as the latter increases. In this note we shall describe two similar situations which occur in real spaces.

The problem in the case of the $K$ plane $^{2}$ has been worked out in Professor Kasner's Seminar in Geometry. ${ }^{3}$ In this plane the length of the curve $y=f(x)$ passing between points of abscissae $x_{1}, x_{2}$, in that order, is given by

$$
l=\int_{x_{1}}^{x_{2}}\left(\frac{d y}{d x}\right)^{-1} d x .
$$

${ }^{1}$ E. Kasner, The ratio of the arc to the chord of an analytic curve need not approach unity, this Bulletin, vol. 21 (1914), pp. 524-531. Similar questions for three dimensions are discussed in E. Kasner, Complex geometry and relativity, theory of the "rac" curvature, Proceedings of the National Academy of Sciences, 1932, p. 267.

${ }^{2}$ Kasner, Trihornometry, a new chapter of conformal geometry, Proceedings of the National Academy of Sciences, vol. 23, p. 346.

${ }^{3} \mathrm{R}$. Coleman, S. Jablon and D. Mittleman obtained the results for the $K$ plane given below. 